\title{
ПСИХОСОМАТИКА
}

УДК 616.89-053.6:616-036.12-055.5/.7

Для цитирования: Катерная Ю.Е. Особенности психического здоровья подростков с отягощенностью соматической патологией у родственников. Сибирский вестник психиатрии и наркологии. 2017; 4 (97): 62-67. https://doi.org/10.26617/18103111-2017-4(97)-62-67

\section{Особенности психического здоровья подростков с отягощенностью соматической патологией у родственников}

\section{Катерная Ю.Е.}

Нижегородская государственная медицинская академия

Россия, 603005, Нижний Новгород, ул. Ульянова, 41

\section{PEЗЮME}

Введение. Наличие тяжелой хронической соматической патологии может приводить к развитию психических (в первую очередь тревожных) расстройств не только у самого пациента, но и членов его семьи. Настоящее исследование было проведено с целью изучения особенностей психического здоровья подростков, родители и/или другие члены семьи которых имели соматическую патологию. Методы. Исследование проведено в форме одномоментного когортного анонимного анкетирования. Участниками исследования являлись 1137 подростков, никогда ранее не обращавшихся за психиатрической и/или наркологической помощью. Результаты. 48,2\% респондентов указали на наличие соматической патологии у родителей и других членов семьи. Сам факт наличия соматической патологии у членов семьи не являлся непосредственной причиной развития психической патологии у подростков. Достоверно более высокий уровень непсихотических психических расстройств был выявлен у респондентов, чьи родственники имели тяжелую соматическую патологию (требовавшую постоянного приема лекарственных препаратов или непосредственно угрожавшую жизни) и/или находившихся в неблагоприятных социальных условиях. К неблагоприятным социальным условиям относились недостаточный материальный уровень, алкогольная и/или наркотическая зависимость у членов семьи. Заключение. Высокий уровень депрессивных и тревожных расстройств среди подростков выявлялся при наличии хронических соматических заболеваний у членов семьи в двух случаях: 1) соматическая патология непосредственно угрожала жизни или требовала постоянного приема лекарственных препаратов, 2) имела место неблагоприятная социальная обстановка. Полученные результаты позволяют сделать вывод об актуальности проведения профилактических мероприятий с целью снижения уровня депрессивных и тревожных нарушений среди школьников, родственники которых имели тяжелую хроническую соматическую патологию.

Ключевые слова: подростки, психические расстройства, соматическая патология, депрессия, тревожные расстройства.

\section{ВВЕДЕНИЕ}

Анализ результатов современных отечественных и зарубежных исследований показывает высокую коморбидность соматической и психической патологии во всех возрастных группах. По мнению ряда авторов, до половины всех жалоб, предъявляемых врачу общего профиля (терапевту и педиатру), относятся к психосоматическому спектру, затрудняют диагностику и лечение основного расстройства $[1,2$, $3,4]$.

С более высокой частотой развитие психических нарушений происходит при наличии тяжелой соматической патологии, а именно хронических, длительно протекающих заболеваний, требующих от больного постоянного приема лекарственных препаратов, регулярных госпитализаций в соматические стационары и пр. Рост психических расстройств наблюдается также в тех случаях, когда соматическая патология существует в форме непосредственно угрожающих жизни состояний. В большинстве случаев наличие подобного рода заболеваний приводит к развитию непсихотических психических нарушений, чаще всего тревожных и депрессивных $[5,6,7,8,9,10]$.
Часть авторов высказывает мнение, что наличие тяжелой хронической соматической патологии приводит к развитию психических расстройств не только у самого пациента, но и членов его семьи. Около пятой части обследованных членов семей пациентов с тяжелыми соматическими заболеваниями имели проявления невротических расстройств, в первую очередь тревожных $[11,12,13]$.

В литературе авторами указывается на ряд причин, приводящих к развитию психических расстройств среди членов семей пациентов с соматическими заболеваниями $[14,15,16]$. В первую очередь считается, что сам факт наличия тяжелой соматической патологии приводит к развитию стрессовой ситуации в семье и, как следствие, появлению реактивных тревожных и депрессивных расстройств среди родственников пациента. Значимую роль играют также дисгармоничные семейные отношения вследствие наличия самого соматического заболевания, необходимости постоянного приема лекарственных препаратов или регулярных госпитализаций. Определенное значение имеют материальные и социальные ограничения вследствие тяжелой соматической патологии у членов семьи $[12,14,15,17]$. 
Таким образом, приведенные выше сведения позволяют считать, что наличие хронических заболеваний способствует развитию непсихотических психических нарушений у членов семьи больного. Однако анализ литературы показал, что развитию тревожных нарушений в подростковом возрасте вследствие соматических заболеваний у родителей и других родственников уделяется мало внимания, что делает изучение данной проблемы актуальным.

Цель исследования - изучение влияния тяжелой соматической патологии у родителей и других членов семьи на психическое здоровье подростков.

\section{МАТЕРИАЛЫ И МЕТОДЫ}

Исследование проводилось в форме одномоментного когортного анкетирования с применением Базисной карты для проведения клиникоэпидемиологических этнокультуральных исследований, которая содержала демографические и социальные данные о респонденте и перечень психопатологических симптомов, объединенных в синдромы в соответствии с критериями Международной классификации болезней 10-го пересмотра и DSMIV. Для оценки суицидального риска была использована Шкала причин для жизни (RFL-40). Анкеты заполнялись респондентами самостоятельно в присутствии исследователя (врача-психиатра).

Участники обследования в большинстве случаев находились в возрасте младше 18 лет и являлись несовершеннолетними, вследствие чего их родителями заполнялось добровольное письменное информированное согласие. Респонденты в возрасте 18 лет и старше самостоятельно принимали решении об участии в исследовании.

Общую выборку составили 1064 подростка в возрасте от 10 до 18 лет, обучавшихся в 7-11-х классах школ различного профиля (общеобразовательных, с углубленным изучением предметов гуманитарного или физико-математического профиля). Лица женского пола составили 52,3\%, мужского пола $-47,7 \%$. В большинстве случаев респонденты были в возрасте 15 лет и старше $(63,1 \%)$.

Контрольная группа была представлена учащимися специализированных учебных центров $(\mathrm{n}=73)$. Данные учебные заведения осуществляли обучение как по программам коррекционных классов, так и по общеобразовательным. Респонденты контрольной группы всегда были в возрасте старше 15 лет, среди них незначительно преобладали девушки $(56,4 \%)$. Учащиеся специализированных учебных центров изначально имели более высокий уровень социальных проблем, таких как низкий материальный уровень семьи, нарушения поведения (вплоть до совершения противоправных действий), употребление алкоголя и/или наркотических препаратов. Обычно данное учебное заведение было вторым или третьим, в котором обучался подросток. Причинами перевода обычно были следующие: слабая заинтересованность в обучении и, как следствие, низкая успеваемость, нарушения поведения, тяжелая соматическая патология, беременность в подростковом возрасте.
В исследовании были задействованы исключительно подростки, никогда ранее не обращавшиеся за психиатрической и/или наркологической помощью и обучавшиеся по общеобразовательным программам.

\section{РЕЗУЛЬТАТЫ И ОБСУЖДЕНИЕ}

Подросткам, принявшим участие в исследовании, было предложено сообщить о наличии тяжелой соматической патологии у родителей и/или других членов семьи, о форме заболевания, необходимости приема лекарственных препаратов, частоте и/или регулярности госпитализаций. Респонденты имели возможность выбрать из списка предложенных заболеваний соответствующее или написать его самостоятельно в поле для замечаний. Учащимся было предложено указать только ту патологию, о которой они достоверно знали на момент проведения исследования, не обращаясь за дополнительной информацией к родителям или другим членам семьи.

Среди участников обследования, заполнивших пункт о соматической патологии у родителей и/или других членов семьи $(52,5 \%$ в общей выборке и 42,5\% в контрольной группе), только 12,5\% отметили формулировку диагноза относительно близко к соответствующей критериям МКБ-10, 64,5\% в форме, позволяющей идентифицировать заболевание (например, «гипертония» вместо «гипертоническая болезнь»). Оставшиеся 23\% выбрали только систему, к которой относилась патология (например, нервная система), или название, которое не позволило его определить с достаточной достоверностью («колики»). Таким образом, в 77\% случаев можно было достоверно определить заболевание, которым страдали родители или другие родственники школьника.

Среди подростков, отметивших достаточно определенную формулировку диагноза, преобладали лица в возрасте 16-18 лет $(65,5 \%)$, чаще всего это были подростки женского пола (62,5\%). Школьники младше 14 лет в подавляющем большинстве случаев, указывая на соматическую патологию, сообщали только о системе, к которой относилась указанная патология, или приводили её бытовое название. Половое распределение среди них было примерно равным (52,2\% лиц женского пола).

Следует отметить, что при наличии одинаковой соматической патологии у ребенка и его родителя достаточно точную формулировку знали большинство подростков $(92,5 \%)$ вне зависимости от пола и возраста.

Таким образом, 38,2\% подростков в общей выборке и $35,2 \%$ в контрольной группе (учащиеся специализированных учебных центров) сообщили о достоверном наличии соматического заболевания у родителей или других членов семьи.

Дополнительно респондентам было предложено указать родственника, у которого выявлялось соматическое заболевание, а также необходимость в приеме лекарственных препаратов и/или госпитализаций. 
Подавляющая часть школьников отметила, у кого из членов семьи имело место соматическое заболевание $(95,6 \%)$. Из них 45,5\% указали о наличии соматической патологии у матери, $40,4 \%$ - у отца, $34,5 \%$ - у бабушки, 32,6\% - у дедушек. В 24,3\% случаев соматическую патологию имели другие родственники. Наличие соматических заболеваний удвух и более членов семьи отметили 22,3\%. О необходимости приема лекарственных средств родственниками, имевшими соматическую патологию, сообщили $34,5 \%$ подростков, $12,3 \%$ - о постоянном систематическом употреблении лекарств, 2,8\% о необходимости регулярных госпитализаций.

Проведенный анализ показал, что как у подростков, обучающихся в различных учебных заведениях, так и в контрольной группе распространенность соматических заболеваний среди членов семьи была примерно одинаковой. Наиболее часто респонденты указывали на наличие у родственников нарушений зрения, гипертонической болезни, аллергических состояний, патологии желудочно-кишечного тракта и частых простудных заболеваний (табл. 1).

Т а б л и ц а

Распространенность соматической патологии

у членов семей среди подростков, обучавшихся в различных учебных заведениях

\begin{tabular}{|l|c|c|c|c|}
\hline \multicolumn{1}{|c|}{ Соматическое заболевание } & 1 & 2 & 3 & 4 \\
\hline Гипертоническая болезнь & $5,2 \%$ & $0 \%$ & $3,1 \%$ & $7,7 \%$ \\
\hline $\begin{array}{l}\text { Язвенная болезнь желудка } \\
\text { и двенадцатиперстной кишки }\end{array}$ & $3,5 \%$ & $2,8 \%$ & $6,1 \%$ & $3,8 \%$ \\
\hline $\begin{array}{l}\text { Хронический гастрит } \\
\text { и гастродуоденит }\end{array}$ & $3,2 \%$ & $2,8 \%$ & $2,6 \%$ & $2,6 \%$ \\
\hline Бронхиальная астма & $2,1 \%$ & $4,2 \%$ & $3,6 \%$ & $2,8 \%$ \\
\hline Сахарный диабет & $5,7 \%$ & $5,6 \%$ & $2,6 \%$ & $3,8 \%$ \\
\hline $\begin{array}{l}\text { Частые простудные заболевания } \\
\text { (чаще 4 раз за год) }\end{array}$ & $6,3 \%$ & $0,9 \%$ & $2,8 \%$ & $8,6 \%$ \\
\hline Вегетососудистая дистония & $2,1 \%$ & $2,8 \%$ & $5,1 \%$ & $5,1 \%$ \\
\hline Аллергические состояния & $5,7 \%$ & $11,1 \%$ & $4,6 \%$ & $5,1 \%$ \\
\hline Неврологические заболевания & $2,9 \%$ & $1,4 \%$ & $7,7 \%$ & $5,1 \%$ \\
\hline Другие соматические заболевания & $1,8 \%$ & $1,4 \%$ & $5,1 \%$ & $3,8 \%$ \\
\hline
\end{tabular}

П р и м е ч а н и е. Место обучения: 1 - общеобразовательные школы, 2 - учебные заведения с углубленным изучением предметов гуманитарного цикла, 3 - учебные заведения с углубленным изучением предметов физико-математического цикла, 4 контрольная группа (специализированный учебный центр).

В дальнейшем изучали взаимосвязь соматической патологии у членов семьи и психических расстройств у подростков. Значимые нарушения психического здоровья у школьников данной группы, за редким исключением, выявлялись только в том случае, если родственники, имевшие тяжелые соматические заболевания, проживали совместно с ними (на одной жилплощади).

Обнаружено, что наличие тяжелой соматической патологии у родителей и/или других членов семьи негативно сказывалось на психологическом состоянии подростков: симптом тревоги («чрезмерная тревога и беспокойство (ожидание плохого) в связи с различными событиями или видами деятельности») имели 75,5\% подростков данной группы в общей выборке и 72,2\% в контрольной группе, «трудности при попытке справиться с тревогой» $-52,2 \%$ и $56,5 \%$ соответственно.
Среди подростков, указавших наличие соматических заболеваний среди членов семьи, наблюдался достоверно более высокий уровень непсихотических психических расстройств (табл. 2).

Т а б ли ц а 2

Частота развития психических расстройств у подростков, имевших членов семьи с соматической патологией

\begin{tabular}{|c|c|c|c|c|}
\hline $\begin{array}{l}\text { Психическое } \\
\text { расстройство }\end{array}$ & 1 & 2 & 3 & 4 \\
\hline $\begin{array}{l}\text { Депрессивный } \\
\text { синдром }\end{array}$ & $\begin{array}{c}20,2 \% \\
\mathrm{C}=0,425 \\
\mathrm{p}=0,0011\end{array}$ & $\begin{array}{c}18,4 \% \\
\mathrm{C}=0,406 \\
\mathrm{p}=0,0001\end{array}$ & $\begin{array}{c}17,2 \% \\
C=0,282 \\
p=0,0258\end{array}$ & $14,07 \%$ \\
\hline $\begin{array}{l}\text { Генерализованное } \\
\text { тревожное рас- } \\
\text { стройство }\end{array}$ & $\begin{array}{c}18,8 \% \\
C=0,402 \\
p=0,0202\end{array}$ & $\begin{array}{c}17,5 \% \\
C=0,442 \\
p=0,0022\end{array}$ & $\begin{array}{c}14,8 \% \\
\mathrm{C}=0,252 \\
\mathrm{p}=0,0124\end{array}$ & $10,6 \%$ \\
\hline $\begin{array}{l}\text { Паническое } \\
\text { расстройство }\end{array}$ & $\begin{array}{c}17,2 \% \\
C=0,422 \\
p=0,0001\end{array}$ & $\begin{array}{c}17,4 \% \\
C=0,454 \\
p=0,0002\end{array}$ & $\begin{array}{c}16,2 \% \\
C=0,226 \\
p=0,0452\end{array}$ & $9,4 \%$ \\
\hline Социальная фобия & $\begin{array}{c}7,8 \% \\
\mathrm{C}=0,284 \\
\mathrm{p}=0,0012\end{array}$ & $\begin{array}{c}7,2 \% \\
C=0,304 \\
p=0,0015\end{array}$ & $\begin{array}{c}6,2 \% \\
C=0,302 \\
p=0,0018\end{array}$ & $3,3 \%$ \\
\hline
\end{tabular}

П р и м е ч а н и е. 1 - Подростки, родственники которых имели соматическую патологию и постоянно принимали лекарственные препараты, 2 - подростки, родственники которых имели соматическую патологию, проявления которой непосредственно угрожали жизни, 3 - подростки, родственники которых имели соматическую патологию, 4 - общая выборка.

В большинстве случаев наблюдался рост тревожных нарушений (генерализованного тревожного расстройства, панического расстройства, фобий). Но результаты проведенного статистического анализа показали, что наличие соматической патологии имеет слабую связь с развитием психических нарушений. Значительный рост показателей психических расстройств (в 2 и более раза в некоторых случаях) связан с другими факторами, такими как дисгармоничные семейные отношения, физическое насилие в семье и соматическая патология у самого подростка. Последний факт был объяснен тем, что около $60 \%$ подростков, указавших на соматическую патологию у родителей или других членов семьи, имели те же заболевания, что и их родители.

Исключением являлись тяжелые соматические заболевания у членов семьи, требовавшие постоянного приема лекарственных препаратов и/или регулярных госпитализаций. В этом случае выявлялась достоверная связь $(\mathrm{p} \leq 0,05)$ между тяжелой соматической патологией у родителей и психическими нарушениями у подростков, а именно генерализованным тревожных расстройством, паническим расстройством и депрессивным состоянием (вне зависимости от конкретного заболевания).

Высокий уровень психических расстройств в сравнении со сверстниками отмечался у подростков, родственники которых имели соматические заболевания с непосредственно угрожающими жизни проявлениями (анафилактический шок, отек Квинке и т.п.). Статистический анализ показал, что имеет место достоверная значимая связь между данными проявлениями соматической патологии и психическими нарушениями, а именно с генерализованным тревожным расстройством и паническим расстройством, в меньшей степени - с депрессией. 
Однако данная закономерность наблюдалась только в том случае, если подросток проживал совместно с лицом, имевшим соматическое заболевание, и это заболевание относилось к первой линии родства (родители, братья и сестры).

Следует отметить, что наличие тяжелой соматической патологии, одинаковой у подростка и родственников первой линии родства и требовавшей постоянного приема лекарственных препаратов и частых госпитализаций, не приводило к повышению уровня тревоги и достоверному увеличению показателей психических расстройств в случае гипертонической болезни и заболеваний желудочнокишечного тракта, а наоборот, приводило к снижению субъективного уровня тревоги. В то же время присутствие угрожающих жизни состояний одновременно у подростка и членов его семьи способствовало достоверному увеличению генерализованого тревожного расстройства и панического расстройства $(\mathrm{p} \leq 0,05)$.

Было выявлено, что при прочих равных условиях значимую роль для развития психической патологии у школьников, родственники которых имели соматические заболевания, играли социальные факторы, а именно состав семьи респондента, финансовый уровень и наличие алкогольной и/или наркотической зависимости среди членов семьи.

Школьники из неполных семей, воспитывавшиеся только матерью или бабушкой и не имевшие материальной поддержки от второго родителя, при наличии хронической соматической патологии достоверно чаще имели генерализованное тревожное расстройство, панические атаки и социофобию (табл. 3). При необходимости постоянного приема лекарственных препаратов или регулярных госпитализаций указанные выше психические нарушения диагностировались значительно чаще в сравнении с общей выборкой $(\mathrm{p} \leq 0,05)$.

Т а б л и ц а 3 Частота психических расстройств у подростков, находившихся в неблагоприятных социальных условиях, члены семьи которых имели соматическую патологию

\begin{tabular}{|c|c|c|c|c|}
\hline $\begin{array}{l}\text { Психическое } \\
\text { расстройство }\end{array}$ & 1 & 2 & 3 & 4 \\
\hline $\begin{array}{l}\text { Депрессивный } \\
\text { синдром }\end{array}$ & $\begin{array}{c}15,2 \% \\
C=0,302 \\
p=0,0025\end{array}$ & $\begin{array}{c}15,4 \% \\
C=0,320 \\
p=0,0021\end{array}$ & $\begin{array}{c}16,2 \% \\
C=0,285 \\
p=0,0021\end{array}$ & $14,07 \%$ \\
\hline $\begin{array}{l}\text { Генерализованное } \\
\text { тревожное рас- } \\
\text { стройство }\end{array}$ & $\begin{array}{c}17,2 \% \\
\mathrm{C}=0,420 \\
\mathrm{p}=0,0012\end{array}$ & $\begin{array}{c}18,8 \% \\
\mathrm{C}=0,462 \\
\mathrm{p}=0,0011\end{array}$ & $\begin{array}{c}19,2 \% \\
C=0,356 \\
p=0,0011\end{array}$ & $10,6 \%$ \\
\hline $\begin{array}{l}\text { Паническое } \\
\text { расстройство }\end{array}$ & $\begin{array}{c}14,2 \% \\
\mathrm{C}=0,426 \\
\mathrm{p}=0,0025\end{array}$ & $\begin{array}{c}16,2 \% \\
C=0,402 \\
p=0,0001\end{array}$ & $\begin{array}{c}18,2 \% \\
\mathrm{C}=0,482 \\
\mathrm{p}=0,0001\end{array}$ & $9,4 \%$ \\
\hline Социальная фобия & $\begin{array}{c}7,8 \% \\
C=0,368 \\
p=0,0014\end{array}$ & $\begin{array}{c}4,4 \% \\
C=0,352 \\
p=0,0042\end{array}$ & $\begin{array}{c}4,8 \% \\
\mathrm{C}=0,320 \\
\mathrm{p}=0,0022\end{array}$ & $3,3 \%$ \\
\hline
\end{tabular}

П р и м е ч а н и е. 1 - Подростки из неполных семей, 2 подростки из семей с низким материальным уровнем, 3 - употребление алкоголя и/или наркотиков родителями, 4 - общая выборка.

Полученные результаты дают возможность сделать предположение, что наличие тяжелого соматического заболевания у лица, в одиночку занимаю- щегося воспитанием и содержанием подростка, вызывало более высокий уровень тревоги у школьников. Данная ситуация может быть объяснена тем, что возможность потери единственного родителя приводит к обстановке постоянного дисстресса и способствует развитию невротической симптоматики у подростка.

Еще одну группу риска составляли респонденты, проживавшие в семьях с выраженными социальными проблемами, такими как недостаточный материальный уровень и алкогольная и/или наркотическая зависимость. В этом случае при наличии тяжелой соматической патологии у родственников выявлялся высокий уровень тревожных расстройств у школьников: частота развития панических атак и генерализованного тревожного расстройства на 6$8 \%$ была выше, чем в общей выборке $(\mathrm{p} \leq 0,05)$.

При сочетании двух указанных выше факторов генерализованное тревожное расстройство и панические атаки развивались чаще на $12-16 \%$ в сравнении с общей выборкой.

Приведенные выше результаты показали, что сам факт наличия соматической патологии, по сути, не является существенным фактором риска для развития психической патологии в подростковом возрасте. В том случае, когда заболевание не несло непосредственной угрозы для жизни или не требовало постоянного приема лекарственных препаратов и/или частых госпитализаций, оно не способствовало развитию дисстресса и психологического дискомфорта, не приводило к развитию психических нарушений среди школьников.

В группе лиц, чьи родственники страдали от соматической патологии, значение имели следующие социальные факторы: неблагоприятная обстановка в семье, низкий материальный уровень и неполная семья (в случае отсутствия материальной помощи от второго родителя). Таким образом, развитие психических расстройств при наличии соматической патологии возникало только при сочетании с изначально неблагоприятными социальными факторами.

\section{ВЫВОДЫ}

Проведенное исследование показало, что 48,2\% подростков в общей выборке и $35,2 \%$ в контрольной группе сообщили о наличии соматических заболеваний у родителей или других членов семьи.

При статистическом анализе полученных результатов было обнаружено, что сам факт присутствия соматической патологии у членов семьи не являлся причиной, непосредственно вызывающей развитие психических нарушений у школьников.

Достоверно более высокий уровень психических расстройств среди подростков диагностировался в том случае, если их родственники имели тяжелые соматические заболевания (требовавшие постоянного приема лекарственных препаратов или представлявшие непосредственную угрозу для жизни). При этом наблюдалось повышение частоты развития депрессивных и тревожных расстройств в сравнении с общей выборкой. 
Вторым фактором, оказывающим влияние на психическое состояние школьников, члены семьи которых имели соматическую патологию, являлась неблагоприятная социальная обстановка. При низком материальном уровне и алкогольной и/или наркотической зависимости у родителей или ближайших родственников происходило достоверное увеличение встречаемости тревожных расстройств среди подростков. Приведенные выше факторы позволяют сделать вывод о необходимости проведения профилактических мероприятий с целью снижения уровня депрессивных и тревожных нарушений среди школьников, родственники которых имели тяжелую хроническую соматическую патологию.

\section{КОНФЛИКТ ИНТЕРЕСОВ}

Автор заявляет об отсутствии конфликта интересов в связи с публикацией данной статьи.

\section{ИСТОЧНИК ФИНАНСИРОВАНИЯ}

Автор заявляет об отсутствии финансирования при проведении исследования.

\section{СООТВЕТСТВИЕ ПРИНЦИПАМ ЭТИКИ}

Работа соответствует этическим стандартам Хельсинской декларации ВМА (протокол заседания Локального этического комитета Нижегородской ГМА № 10 от 27 октября 2014 г., тема диссертационного исследования утверждена на Ученом совете Нижегородской ГМА, протокол № 169 от 1 декабря 2014 г.).

\section{ЛИТЕРАТУРА}

1. Собенников В.С., Белялов Ф.И. Соматизация и психосоматические расстройства. Иркутск: РИО ИГИУВ, 2010: 230.

2. Friman P., Handwerc M., Swearer S. et al. Do Children with primary nocturnal enuresis have clinically significant problems? Arch. Pediatr. Adolesc. Med. 1998; 152: 573-579.

3. Kisely S., Goldberg D. Physical and psychiatric comorbidity in general practice. British Journal Psychiatry. 1996; 44 (2): 236-242.

4. Spady D.W., Schopflocher D., Svenson L. et al. Medical and Psyctiatric Comorbidity and Health Care Use Among Children 6 to 17 Years Old. Arch. Pediatr. Adolesc. Med. 2005; 159: 231-237.

5. Николаев Е.Л. Здоровье и благополучие родственника больного: только ли генетические риски? Вестник психиатрии и психологии Чувашии. 2016; 2: 6-12.

6. Мальцева М.Н., Шмонин А.А., Мельникова Е.В. Феномен «семейной инвалидности», или депрессия у родственников, ухаживающих за пациентом с инвалидностью. Ученые запис- ки Санкт-Петербургского государственного медищинского университета им. акад. И.П. Павлова. 2016; 23 (3): 40-43.

7. Созинова Е.В., Глозман Ж.М. Качество жизни родственников пациентов, находящихся на ранних стадиях болезни Паркинсона. Вестник Московского университета. Серия 14: Психология. 2009; 3: 88-95.

8. Гантман М.В. Когнитивные и некогнитивные психические расстройства у лиц пожилого возраста, ухаживающих за пациентами с болезнью Альцгеймера. Журнал неврологии и психиатрии им. С.С. Корсакова. 2014; 114 (6): 49-54.

9. Полищук Ю.И., Гурвич В.Б., Летникова 3.В., Баскакова С.А. Клинические особенности, причины и содержание тревожных расстройств невротического уровня в позднем возрасте. Психическое здоровье. 2009; 10: 21-25.

10. Горячева Е. Е. Структура депрессивных расстройств у пациентов с функциональной патологией желудочно-кишечного тракта. Медииинский альманах. 2015; 1 (36): 30-33.

11. Лександрова С.С., Грынчарова Г.Г. Проблемы и тревоги у родственников пациентов, обслуживаемых в болгарских хосписах. Медииинский вестник Юга России. 2010; 1: 17-20.

12. Захаров О.П., Куташов В.А. Нарушение адаптации у родственников пациентов, перенесших острое нарушение мозгового кровообращения. Вестник неврологии, психиатрии и нейрохирургии. 2015; 10: 65-69.

13. Сучкова С.П., Румянцева С.А. Психосоматические проявления тревоги и депрессии и их возможные последствия у родственников больных в условиях многопрофильного стационара. Нервные болезни. 2009; 2: 30-32.

14. Белинская Е.П., Сангова С. Болезнь как трудная жизненная ситуация: особенности стратегий совладания родственников тяжелобольных. Психологические исследования. 2015; 8 (42): 8 URL: http://psystudy.ru (Дата обращения: 25.05.2017).

15. Гарганеева Н. П., Белокрылова М. Ф. Основные симптомокомплексы и условия формирования невротических и аффективных расстройств у больных с сердечно-сосудистыми заболеваниями. Сибирский медицинский журнал. 2009; 4-2: 11-17.

16. Куприянова И.Е, Дашиева Б.А., Карауш И.С. Клиникопсихологические, биологические и социальные факторы риска развития психопатологических нарушений у детей с ограниченными возможностями здоровья. Сибирский вестник психиатрии и наркологии. 2012; 3 (72): 36-40.

17. Адриянова Е.А., Аранович И.Ю., Кураева Д.А. Взаимосвязь отношения к болезни и детско-родительских отношений у подростков с бронхиальной астмой. Сборники конференций НИЦ Социосфера. 2013; 21: 50-52.

Поступила в редакцию 5.07.2017 Утверждена к печати 30.10.2017

Катерная Юлия Евгеньевна, аспирант кафедры психиатрии и медицинской психологии.

Катерная Юлия Евгеньевна, katernaya.yulia @ gmail.com

УДК 616.89-053.6:616-036.12-055.5/.7

For citation: Katernaya J.E. Mental health of the adolescents whose relatives had the somatic pathology. Siberian Herald of Psychiatry and Addiction Psychiatry. 2017; 4 (97): 60-65. https://doi.org/10.26617/1810-3111-2017-4(97)-60-65

\section{Mental health of the adolescents whose relatives had the somatic pathology Katernaya J.E.}

Nizhny Novgorod State Medical Academy

Ulyanov Street 41, 603005, Nizhniy Novgorod, Russian Federation

\section{ABSTRACT}

Introduction. The presence of severe chronic somatic pathology can lead to the development of mental (primarily anxious) disorders not only in the patient himself, but also in his family. Methods. The study was conducted in the form of a one-stage cohort anonymous questionnaire. The present research was conducted to study the characteristics of mental health of adolescents, parents and/or other members of the family which had somatic pathology. Participants in the study were adolescents $(n=1137)$, who had 
never previously applied psychiatric and/or drug treatment. Results. $48.2 \%$ of respondents indicated the presence of somatic pathology in parents and other family members. The conducted study showed that the fact of somatic pathology in family members was not the immediate cause of the development of mental pathology in schoolchildren. The significantly higher level of nonpsychotic mental disorders was revealed that of the respondents whose relatives had severe somatic pathology (requiring permanent medication or immediately life-threatening) and/or were in adverse social conditions. Adverse social conditions included insufficient material level, alcohol and / or drug dependence among family members. Conclusion. The high level of depressive and anxiety disorders among adolescents was detected in the presence of chronic somatic diseases in family members in two cases: 1) somatic pathology directly threatened life or required constant intake of medications, 2) there was an adverse social situation. The results obtained showed the relevance of preventive measures to reduce the level of depressive and anxiety disorders among schoolchildren whose relatives had severe, chronic somatic pathology.

Keywords: adolescents, mental disorders, somatic pathology, depression, anxiety disorder.

\section{REFERENCES}

1. Sobennikov V.S., Belyalov F.I. Somatizatsiya i psihosomaticheskie rasstroystva [Somatization and psychosomatic disorders]. Irkutsk: RIO IGIUV, 2010: 230 In Russian).

2. Friman P., Handwerc M., Swearer S. et al. Do Children with primary nocturnal enuresis have clinically significant problems? Arch. Pediatr. Adolesc. Med. 1998; 152: 573-579.

3. Kisely S., Goldberg D. Physical and psychiatric comorbidity in general practice. British Journal of Psychiatry. 1996; 44 (2): 236-242.

4. Spady D.W., Schopflocher D., Svenson L. et al. Medical and Psyctiatric Comorbidity and Health Care Use Among Children 6 to 17 Years Old. Arch. Pediatr. Adolesc. Med. 2005; 159: 231237.

5. Nikolaev E.L. Zdorove i blagopoluchie rodstvennika bolnogo: tolko li geneticheskie riski? [Health and well-being of a patient's relative: are there only genetic risks?]. Vestnik psihiatrii $i$ psihologii Chuvashii - Bulletin of Psychiatry and Psychology of Chuvashia. 2016; 2: 6-12 (in Russian).

6. Maltseva M.N., Shmonin A.A., Melnikova E.V. Fenomen «semeynoy invalidnosti», ili depressiya u rodstvennikov, uhazhivayuschih za patsientom $\mathrm{s}$ invalidnostyu [Phenomenon of "family disability" or depression in relatives caring about the patient with disability]. Uchenyie zapiski Sankt-Peterburgskogo gosudarstvennogo meditsinskogo universiteta im. akad. I.P. Pavlova - Scientific Notes of Saint-Petersburg State Medical University. 2016; 23 (3): 40-43 (in Russian).

7. Sozinova E.V., Glozman Zh.M. Kachestvo zhizni rodstvennikov patsientov, nahodyaschihsya na rannih stadiyah bolezn Parkinsona [Quality of life of relatives of patients at early stages of Parkinson's disease]. Vestnik Moskovskogo universiteta. Seriya 14: Psihologiya - Bulletin of Moscow University. Series 14: Psychology. 2009; 3: 88-95 (in Russian).

8. Gantman M.V. Kognitivnyie i nekognitivnyie psihicheskie rasstroystva u lits pozhilogo vozrasta, uhazhivayuschih za patsientami s boleznyu Altsgeymera [Mental disorders of cognitive and non-cognitive spectrum in the elderly caregivers of the patients with Alzheimer"s disease]. Zhurnal nevrologii i psihiatrii im. S.S. Korsakova - S.S. Korsakov Journal of Neurology and Psychiatry. 2014; 114 (6): 49-54 (in Russian).

9. Polischuk Yu.I., Gurvich V.B., Letnikova Z.V., Baskakova S.A. Klinicheskie osobennosti, prichinyi i soderzhanie trevozhnyih rasstroystv nevroticheskogo urovnya v pozdnem vozraste [Clinical features, reasons and contents of neurotic anxiety disorders in old age persons]. Psihicheskoe zdorove - Mental Health. 2009; 10: 21-25 (in Russian).

10. Goryacheva E. E. Struktura depressivnyih rasstroystv u patsientov $\mathrm{s}$ funktsionalnoy patologiey zheludochnokishechnogo trakta [Structure of depressive disorders in the case of patients with functional gastrointestinal pathology]. Med- itsinskiy almanah - Medical Literary Miscellany. 2015; 1 (36): 30-33 (in Russian).

11. Leksandrova S.S., Gryincharova G.G. Problemyi i trevogi u rodstvennikov patsientov, obsluzhivaemyih $\mathrm{v}$ bolgarskih hospisah [problems and alarms of the patients relatives served in the Bulgarian hospices]. Meditsinskiy vestnik Yuga Rossii - Medical Bulletin of the South of Russia. 2010; 1: 17-20 (in Russian).

12. Zakharov O.P., Kutashov V.A. Narushenie adaptatsii u rodstvennikov patsientov, perenesshih ostroe narushenie mozgovogo krovoobrascheniya [Trouble adapting relatives whose patients suffered acute ischemic stroke]. Vestnik nevrologii, psihiatrii $i$ neyrohirurgii - Bulletin of Neurology, Psychiatry and Neurosurgery. 2015; 10: 65-69 (in Russian).

13. Suchkova S.P., Rumyantseva S.A. Psihosomaticheskie proyavleniya trevogi i depressii i ih vozmozhnyie posledstviya u rodstvennikov bolnyih v usloviyah mnogoprofilnogo sta-tsionara [Psychosomatic manifestations of anxiety and depression and their possible consequences in relatives of patients in the conditions of multi-profile hospital]. Nervnyie bolezni-Nervous Diseases. 2009; 2: 30-32 (in Russian).

14. Belinskaya E.P., Sangova S. Bolezn kak trudnaya zhiznennaya situatsiya: osobennosti strategiy sovladaniya rodstvennikov tyazhelobolnyih [Diseases as a difficult life situation; features of strategy of coping of relatives of hard-to-cure patients] Psihologicheskie issledovaniya - Psychological Research. 2015; 8 (42): 8 URL: http://psystudy.ru (Data obrascheniya: 25.05.2017) (in Russian).

15. Garganeeva N. P., Belokrylova M. F. Osnovnyie simptomokompleksyi i usloviya formirovaniya nevroticheskih i affektivnyih rasstroystv $\mathrm{u}$ bolnyih $\mathrm{s}$ serdechno-sosudistyimi zabolevaniyami [Basic symptom complexes and conditions of forming neurotic and affective disorders in patients having cardiovascular diseases]. Sibirskiy meditsinskiy zhurnal - Siberian Medical Journal. 2009; 4-2: 11-17 (in Russian).

16. Kupriyanova I.E, Dashieva B.A., Karaush I.S. Klinikopsihologicheskie, biologicheskie i sotsialnyie faktoryi riska razvitiya psihopatologicheskih narusheniy u detey s ogranichennyimi vozmozhnostyami zdorovya [Clinical-psychological, biological and social risk factors of development of psychopathological disturbances in children with disabilities]. Sibirskiy vestnik psihiatrii $i$ narkologii - Siberian Herald of Psychiatry and Addiction Psychiatry. 2012; 3 (72): 36-40 (in Russian).

17. Adriyanova E.A., Aranovich I.Yu., Kuraeva D.A. Vzaimosvyaz otnosheniya $\mathrm{k}$ bolezni i detsko-roditelskih otnosheniy u podrostkov s bronhialnoy astmoy [Interrelationship of attitude towards the illness and child-parent relations with bronchial asthma]. Sborniki konferentsiy NITs Sotsiosfera. 2013; 21: 50-52 (in Russian).

Katernaya Julia E., postgraduate student of Psychiatry and Medical Psychology Department, Nizhny Novgorod State Medical Academy, Nizhny Novgorod, Russian Federation. 